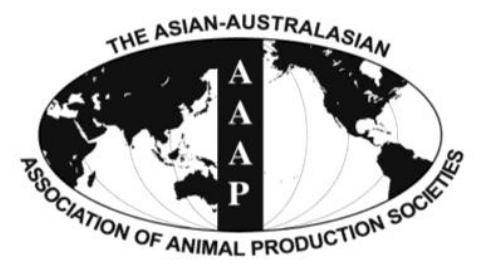

Asian-Aust. J. Anim. Sci.

Vol. 25, No. 4 : 486 - 495

April 2012

www.ajas.info

http://dx.doi.org/10.5713/ajas.2011.11325

\title{
Effect of Dietary Cation-Anion Difference during Prepartum and Postpartum Periods on Performance, Blood and Urine Minerals Status of Holstein Dairy Cow
}

\author{
A. Razzaghi*, H. Aliarabi ${ }^{1}$, M. M. Tabatabaei ${ }^{1}$, A. A. Saki ${ }^{1}$, R. Valizadeh and P. Zamani ${ }^{1}$ \\ Department of Animal Science, Ferdowsi University of Mashhad, P.O.Box 91775-1163, Mashhad, Iran
}

\begin{abstract}
Twenty four periparturient cows were used to determine the effects of DCAD on acid-base balance, plasma and urine mineral concentrations, health status, and subsequent lactation performance. Each group of 12 cows received either a diet containing -100 DCAD or +100 DCAD for 21 d prepartum. Both anionic and cationic groups were divided into two groups, one received a +200 DCAD and the other +400 DCAD diet for $60 \mathrm{~d}$ postpartum. Prepartum reduction of DCAD decreased DMI, urinary and blood pH, urinary concentrations of $\mathrm{Na}$ or $\mathrm{K}$ and increased plasma and urinary $\mathrm{Ca}, \mathrm{Mg}, \mathrm{Cl}$ and $\mathrm{S}$. Also cows fed -100 DCAD diet consumed the most dry matter in the first $60 \mathrm{~d}$ after calving. Postpartum +400 DCAD increased milk fat and total solid percentages, urinary and blood $\mathrm{pH}$ and urinary $\mathrm{Na}$ and $\mathrm{K}$ concentrations, but urinary $\mathrm{Ca}, \mathrm{P}, \mathrm{Cl}$ and $\mathrm{S}$ contents decreased. Greater DMI, FCM yields were observed in cows fed a diet of +400 DCAD than +200 DCAD. No case of milk fever occurred for any diets but feeding with a negative DCAD diet reduced placenta expulsion time. In conclusion, feeding negative DCAD in late gestation period and high DCAD in early lactation improves performance and productivity of dairy cows. (Key Words : Dietary Cation-anion Difference, Lactation, Acid-base Balance, Calcium, Health Status, Dairy Cow)
\end{abstract}

\section{INTRODUCTION}

Besides tremendous changes in energy and protein flux around the time of calving, periparturient cows also experience large changes in mineral element dynamics (Horst et al., 1997). A key component of mineral metabolism in these cows is preventing hypocalcemia, which reduces dry matter intake (Hansen et al., 2003) and increases the risk of metabolic disorders (Curtis et al., 1983).

Hypocalcemia is a particular concern in the newly calved cow, where the sudden demand for calcium at the onset of lactation severely tests the calcium homeostatic capabilities of the animal (Goff, 2008). Hypocalcemia increases the risk of cows getting other diseases. Hypocalcemia is a predisposing factor for dystocia, prolapsed uterus, retained placenta, and early metritis (Grohn et al., 1989; DeGaris and Lean, 2008). Dietary

\footnotetext{
* Corresponding Author : Ali. Razzaghi. Tel : +98-511-8788494, Fax : +98-511-8788494, E-mail : arazzaghi88@gmail.com

${ }^{1}$ Department of Animal science, Bu-Ali Sina University, Hamedan, Iran.

Submitted Sept. 12, 2011; Accepted Nov. 9, 2011; Revised Nov. 14, 2011
}

cation-anion difference has a role in animal productivity and health via its influence on the acid-base balance and calcium metabolism in the animal that often become 'broken' in dairy cows (Sanchez, 2003). Reducing DCAD by increasing dietary acidity or employing anionic salts has been efficacious and cost effective in the prophylaxis of hypocalcemia (Chan et al., 2006). High concentrations of dietary anionic salts cause an influx of negatively charged ions systemically, leading to increased hydrogen ion concentration to maintain electroneutrality. Increased hydrogen ion concentration induces a mild metabolic acidosis. Acidogenic diets are hypothesized to increase bone resorption, blood $\mathrm{Ca}$ and intestinal $\mathrm{Ca}$ absorption (Horst et al., 1997).

The prevalence of hypocalcemia is as high as $70 \%$ for multiparous cows, although only $8 \%$ exhibited clinical hypocalcemia (Beede, 1995) that lowers the $16 \%$ yearly milk yield (Block, 1984). Feeding low DCAD during the 3 to $4 \mathrm{wk}$ before calving had beneficial effects on systemic acid-base status, calcium metabolism, prepartum health and also postpartum productive performance (Horst et al., 1994). However, feeding negative DCAD to periparturient dairy cows proved a useful nutritional practice, it enhanced blood 
calcium and postpartum milk production (Block, 1984; Beede et al., 1992; Moore et al., 2000). The recent advances in mineral nutrition demonstrated that cows fed higher DCAD level produced more milk during early lactation (Sanchez, 2003; Hu and Murphy, 2004; Hu et al., 2007a). The potential effect of DCAD on lactating dairy cows has also been explored, and results indicates that DCAD and production are related possibly through acid-base regulation (Sanchez and Beede, 1994; Hu and Murphy, 2004). About $11 \%$ dry matter intake (DMI) and 9\% milk yield were increased in early lactating cows fed +200 vs. -100 DCAD diet (Tucker et al., 1988).

In addition, manipulating DCAD might benefit lactating dairy cows immediately after calving to about $50 \mathrm{~d}$ postpartum (Chan et al., 2005; Hu et al., 2007b).

The objectives of this study were to determine the interaction effects of prepartum and postpartum diets with different levels of DCAD on plasma and urine concentration of mineral elements, acid-base balance, calcium homeostasis, DMI, milk production and health status in dairy cows.

\section{MATERIALS AND METHODS}

\section{Experimental design and animal care}

The trial consisted a $21 \mathrm{~d}$ prepartum phase followed by a 63 d postpartum phase. Twenty-four multiparous Holstein cows were randomly allocated to a high DCAD $(+100$ $\mathrm{mEq} / \mathrm{kg} \mathrm{DM} ; \mathrm{n}=12)$ or low DCAD $(-100 \mathrm{mEq} / \mathrm{kg} \mathrm{DM} ; \mathrm{n}=$ 12) 3 wk before calving. Anionic salt was added to reduce DCAD. After calving, the cows in each group were reallocated to receive dietary DCADs of +200 or +400 $\mathrm{mEq} / \mathrm{kg} \mathrm{DM}$ (6 cows per group) creating a split-plot in time design with a $2 \times 2$ factorial arrangement. All diets were formulated according to NRC (2001) recommendations for periparturient and early lactation Holstein cows $(\mathrm{DCAD}=$ $(\mathrm{Na}+\mathrm{K}+0.15 \mathrm{Ca}+0.15 \mathrm{Mg})-(\mathrm{Cl}+0.6 \mathrm{~S}+0.5 \mathrm{P}) / \mathrm{kg}$ of $\mathrm{DM})$ (Table 1).

Cows were housed in individual pens and milked three times a day at 04:00, 12:00 and 20:00 h. Water was available ad libitum. DCAD was altered using $\mathrm{CaCl}_{2}$, $\mathrm{NH}_{4} \mathrm{Cl}, \mathrm{MgSO}_{4}$ and $\mathrm{CaSO}_{4}$ or $\mathrm{K}_{2} \mathrm{CO}_{3}$ and $\mathrm{NaHCO}_{3}$ salts in diets. Feed given to cow at 0900 and $2100 \mathrm{~h}$ before calving and at 0500,1400 and $2100 \mathrm{~h}$ after calving.

\section{Sample collection and analysis}

Feed intake of each cow was recorded on d 15, 10, 5 and 2 prepartum and on weekly intervals postpartum. The prepartum or postpartum diet samples, were composited for analysis of DM, CP, EE, Ca, and P (AOAC, 1990) and feed NDF and ADF were analyzed according to the method initially described by Van Soest et al. (1991). The Na, K and
Table 1. Ingredients and chemical composition of diets for dairy cows in pre and post-partum periods

\begin{tabular}{|c|c|c|c|c|}
\hline & \multicolumn{4}{|c|}{ DCAD (mEq/kg DM) } \\
\hline & -100 & +100 & +200 & +400 \\
\hline Ingredients (\% DM) & \multicolumn{2}{|c|}{ Prepartum } & \multicolumn{2}{|c|}{ Postpartum } \\
\hline Alfalfa & 40.07 & 39.90 & 23.96 & 23.82 \\
\hline Corn silage & 28.98 & 28.54 & 14.36 & 14.29 \\
\hline Concentrate mixture $^{1}$ & 28.18 & 29.68 & - & - \\
\hline Beet pulp & 1.13 & 1.67 & - & - \\
\hline Anionic salts ${ }^{2}$ & 1.64 & 0.21 & - & - \\
\hline Concentrate mixture & - & & $61.68^{3}$ & $61.89^{4}$ \\
\hline \multicolumn{5}{|c|}{ Nutrient levels (\% DM) } \\
\hline $\mathrm{NE}_{\mathrm{L}}$ & 1.31 & 1.33 & 1.58 & 1.57 \\
\hline $\mathrm{CP}$ & 13 & 13.2 & 18.4 & 18.5 \\
\hline $\mathrm{NDF}$ & 48.7 & 48.3 & 34.8 & 34.3 \\
\hline $\mathrm{ADF}$ & 27.2 & 27.5 & 19 & 18.9 \\
\hline $\mathrm{Ca}$ & 0.76 & 0.48 & 0.82 & 0.82 \\
\hline $\mathrm{Mg}$ & 0.31 & 0.23 & 0.29 & 0.29 \\
\hline $\mathrm{P}$ & 0.25 & 0.26 & 0.41 & 0.41 \\
\hline $\mathrm{Na}$ & 0.21 & 0.21 & 0.49 & 0.78 \\
\hline $\mathrm{K}$ & 1.19 & 1.20 & 1.14 & 1.42 \\
\hline $\mathrm{Cl}$ & 0.85 & 0.56 & 0.53 & 0.53 \\
\hline $\mathrm{S}$ & 0.42 & 0.22 & 0.24 & 0.24 \\
\hline $\begin{array}{l}\mathrm{DCAD}^{5} \\
(\mathrm{mEq} / \mathrm{kg} \mathrm{DM})\end{array}$ & -108 & +106 & +206 & +404 \\
\hline
\end{tabular}

${ }^{1}$ Concentrate composition (\%): barley, 20.5; corn, 20.5; wheat, 4.5; wheat barn, 28.5; cotton meal, 19; soybean meal, 6; premix 1, 1 .

${ }^{2}$ Ingredients: $\mathrm{NH}_{4} \mathrm{Cl}, \mathrm{CaCl}_{2}, \mathrm{MgSO}_{4}, \mathrm{CaSO}_{4}$ (each $0.41 \%$ for anionic diet).

${ }^{3}$ Composition (for 61.68\%): barley, 13; wheat, 3.78; corn, 12.04; wheat barn, 8.71; cotton meal, 12.04; soybean meal, 7.55; fat calcium soaps, $1.72 ; \mathrm{NaHCO}_{3}, 0.68 ; \mathrm{CaCO}_{3}, 1 ; \mathrm{MgO}, 0.18$; salt, 0.49 ; premix 2, 0.49 .

${ }^{4}$ Composition (for 61.89\%): barley, 12.51; wheat, 2.87; corn, 11.74; wheat barn, 8.30; cotton meal, 12.33; soybean meal, 8.01; fat calcium soaps, 1.71; $\mathrm{NaHCO}_{3}, 1.77 ; \mathrm{K}_{2} \mathrm{CO}_{3}, 0.49 ; \mathrm{CaCO}_{3}, 1 ; \mathrm{MgO}, 0.18$; salt, 0.49; premix 2, 0.49 .

Actually determined dietary cation-anion difference $(\mathrm{Na}+\mathrm{K}+0.15$ $\mathrm{Ca}+0.15 \mathrm{Mg})-(\mathrm{Cl}+0.6 \mathrm{~S}+0.5 \mathrm{P})$.

$\mathrm{Mg}$ contents were measured using atomic absorption spectrophotometry (GBC-3000, Australia), Chlorine was assessed via a potentiometer; $\mathrm{P}$ and $\mathrm{S}$ were detected using spectronic (Spectronic 6300, Australia).

A total of 4 urine samples were collected about $4 \mathrm{~h}$ after the morning feeding on $\mathrm{d} 2$ and 12 prepartum and postpartum. Midstream urine was collected in plastic containers and Urine samples (40-ml for each cow) were stored at $-20^{\circ} \mathrm{C}$ till later assay of mineral contents. Likewise, ten-ml of blood samples were taken from subcutaneous abdominal vein, using heparinized plastic syringes, $4 \mathrm{~h}$ after the morning feeding on $\mathrm{d} 2$ and 12 pre-calving; on $\mathrm{d} 0$ at calving; and on d 2 and 12 postpartum to obtain plasma via centrifugation at 3,000 rpm for $20 \mathrm{~min}$. The plasma was 
stored at $-20^{\circ} \mathrm{C}$ until later analyzed for minerals. Urine and blood $\mathrm{pH}$ on $\mathrm{d} 2$ and 12 prepartum and postpartum was measured immediately after sampling using a hand-held $\mathrm{pH}$ meter (HANNA-210, Italy).

Clinical hypocalcemia was considered if the cow was recumbent and plasma total $\mathrm{Ca}$ concentration was $<5.5$ $\mathrm{mg} / \mathrm{dl}$, and occurrence of subclinical hypocalcemia was established by blood $\mathrm{Ca}$ concentrations below $7.5 \mathrm{mg} / \mathrm{dl}$ at any time during the experimental period (Goff and Horst, 1997). Retained placenta was recorded when the placenta membrane remained in the uterus for $24 \mathrm{~h}$ or longer postpartum (Kelton et al., 1998). Udder edema was recorded if the udder became swollen and the teats became flushed. Mastitis, metritis, endometritis and ketosis were monitored by Veterinarian. Milk production was recored on d 15, 30, 45 and 60. Milk samples from three milkings were collected relative to production on 15 and 30 DIM and analysed for fat, protein, lactose, SNF and TS concentration using Milko-scan (Foss-605, Denmark).

\section{Statistical analysis}

The general linear model procedure of SAS software system (2004) was used to analyze data. All data associated with prepartum cows were analyzed according to the model of a completely randomized design:

$$
\mathrm{Y}_{\mathrm{ij}}=\mu+\mathrm{T}_{\mathrm{i}}+\mathrm{e}_{\mathrm{ij}}
$$

Where $\mu=$ overall mean; $T_{i}=$ effect of treatment $I(i=1$, $2) ; \mathrm{e}_{\mathrm{ij}}=$ error term.

All data related to postpartum cows, except Ca and DMI were analyzed with the GLM procedure (SAS, 2004) according to the model of a split-plot in time design with a $2 \times 2$ factorial arrangement:

$$
\mathrm{Y}_{\mathrm{ijl}}=\mu+\mathrm{A}_{\mathrm{i}}+\mathrm{B}_{\mathrm{j}}+\mathrm{AB}_{\mathrm{ij}}+\mathrm{Ea}_{\mathrm{ijk}}+\mathrm{T}_{\mathrm{l}}+\mathrm{AT}_{\mathrm{il}}+\mathrm{BT}_{\mathrm{jl}}+\mathrm{ABT}_{\mathrm{ijl}}+\mathrm{Eb}_{\mathrm{ijl}}
$$

Where $\mu=$ overall mean; $A_{i}=$ effect of prepartum DCADs; $B_{j}=$ effect of postpartum DCADs; $A_{\mathrm{ij}}=$ effect of interaction between DCAD levels in pre-calving and postpartum; $\mathrm{Ea}_{\mathrm{ijk}}=$ main error; $\mathrm{T}_{1}=$ effect of time; $\mathrm{AT}_{\mathrm{il}}=$ effect of interaction between time and prepartum DCADs; $\mathrm{BT}_{\mathrm{jl}}=$ effect of interaction between time and postpartum DCADs; $\mathrm{ABT}_{\mathrm{ijl}}=$ effects of interaction between time and prepartum and postpartum DCADs; $\mathrm{Eb}_{\mathrm{ijl}}=$ sub-error.

The model used in this study for plasma calcium on $\mathrm{d} 0$ at calving and on d 2 and 12 postpartum and dry matter intake on first 9 wk postpartum was (interactions between treatment and time were significance):

$$
Y_{i j k}=\mu+A_{i}+B_{j}+A B_{i j}+e_{i j k}
$$

Where $\mu=$ overall mean; $A_{i}=$ effect of prepartum DCADs; $B_{j}=$ effect of postpartum DCADs; $A_{i j}=$ effect of interaction between DCAD levels in pre-calving and postpartum; $\mathrm{e}_{\mathrm{ijk}}=$ error term.

The health status was processed by Chi-square test. Duncan's multiple range test was used to examine means. A statistically significant difference was noted unless $\mathrm{p}<0.05$.

\section{RESULTS}

\section{Fluid acid-base balance}

Urinary and blood $\mathrm{pH}$ values were lowest $(\mathrm{p}<0.01)$, in prepartum cows receiving DCAD of $-100 \mathrm{mEq} / \mathrm{kg} \mathrm{DM}$ and highest in cows receiving $+100 \mathrm{mEq} / \mathrm{kg}$ DM (Table 2). Dairy cows fed the +200 - and +400 - DCAD postpartum showed the lowest and highest urine and blood $\mathrm{pH}$ values respectively $(\mathrm{p}<0.01)$. However, urine and blood $\mathrm{pH}$ of lactating cows were not affected by prepartum DCAD diet level (Table 6).

\section{Plasma mineral concentration}

Feeding -100 DCAD diet resulted in higher $(\mathrm{p}<0.01)$ plasma $\mathrm{Ca}$ in periparturient cows than the positive DCAD diet $(+100 \mathrm{mEq} / \mathrm{kg} \mathrm{DM})$. A similar trend was recorded for plasma $\mathrm{Mg}, \mathrm{Cl}$ and $\mathrm{S}$ concentrations. Alteration in prepartum DCAD did not affect the plasma $\mathrm{P}, \mathrm{Na}$ and $\mathrm{K}$ concentrations significantly (Table 2).

Plasma Ca was significantly affected by time, with the nadir on $\mathrm{d} 1$ relative to the higher values on $\mathrm{d} 2$ and 12 postpartum (Table 3). At calving, greater plasma $\mathrm{Ca}$ was observed for -100 DCAD diet compared to +100 DCAD diet $(\mathrm{p}<0.01)$, but no significant difference in postpartum DCAD levels was observed for plasma $\mathrm{Ca}$ on $\mathrm{d} 2$ and 12. Increased plasma $\mathrm{Na}$ and $\mathrm{K}$ concentrations $(\mathrm{p}<0.01)$ were noticed in lactating dairy cows fed +400 DCAD compared to those fed +200 DCAD concentration (Table 4). In early lactation, plasma $\mathrm{Mg}, \mathrm{P}, \mathrm{Cl}$ and $\mathrm{S}$ contents did not differ due to prepartum and postpartum DCAD alteration.

\section{Urine mineral concentration}

Concentrations of $\mathrm{Ca}, \mathrm{Mg}, \mathrm{Cl}$ and $\mathrm{S}$ in urine were higher $(\mathrm{p}<0.01)$ for cows fed -100 DCAD than cows fed +100 DCAD on d 2 and 12 prepartum (Table 2). However, cows fed +100 DCAD had higher $\mathrm{Na}$ and $\mathrm{K}$ contents in urine than cows fed $-100 \mathrm{DCAD}$ at $\mathrm{d} 2$ and 12 pre-calving.

A significant increase $(\mathrm{p}<0.01)$ in urinary $\mathrm{Ca}, \mathrm{P}, \mathrm{Cl}$ and $S$ was recorded in cows fed +200 compared to +400 DCAD diet (Table 5). Urinary $\mathrm{K}$ and $\mathrm{Na}$ contents tended to decrease with decreasing DCAD in early lactation. $\mathrm{Mg}$ concentration remained unaltered due to DCAD alteration across all diets. Urine mineral contents were not affected by dietary prepartum DCAD levels in early lactation cows. 
Table 2. The effects of DCAD on fluid acid-base balance, plasma and urinary mineral concentrations and DMI in periparturient dairy cows

\begin{tabular}{|c|c|c|c|c|c|}
\hline & \multicolumn{4}{|c|}{$\mathrm{DCAD}^{1}(\mathrm{mEq} / \mathrm{kg} \mathrm{DM})$} & \multirow{4}{*}{ SEM } \\
\hline & \multicolumn{2}{|c|}{-100} & \multicolumn{2}{|c|}{+100} & \\
\hline & \multicolumn{4}{|c|}{ Day (pre-calving) } & \\
\hline & 2 & 12 & 2 & 12 & \\
\hline \multicolumn{6}{|l|}{ Urinary $^{2}$} \\
\hline $\mathrm{pH}$ & $6.66^{\mathrm{b}}$ & $6.69^{\mathrm{b}}$ & $7.77^{\mathrm{a}}$ & $7.81^{\mathrm{a}}$ & 0.04 \\
\hline Calcium (mg/d1) & $16.23^{\mathrm{a}}$ & $16.22^{\mathrm{a}}$ & $9.07^{\mathrm{b}}$ & $9.06^{\mathrm{b}}$ & 0.43 \\
\hline Magnesium (mg/d1) & $28.68^{\mathrm{a}}$ & $28.67^{\mathrm{a}}$ & $18.55^{\mathrm{b}}$ & $18.53^{\mathrm{b}}$ & 0.21 \\
\hline Phosphorus (mg/d1) & 1.61 & 1.61 & 1.53 & 1.52 & 0.04 \\
\hline Sodium $(\mathrm{mEq} / \mathrm{L})$ & $94.68^{\mathrm{b}}$ & $94.67^{\mathrm{b}}$ & $125.39^{\mathrm{a}}$ & $125.37^{\mathrm{a}}$ & 0.59 \\
\hline Potassium (mEq/L) & $43.49^{\mathrm{b}}$ & $43.48^{\mathrm{b}}$ & $46.17^{\mathrm{a}}$ & $46.17^{\mathrm{a}}$ & 0.19 \\
\hline Chloride (mEq/L) & $246.24^{\mathrm{a}}$ & $246.23^{\mathrm{a}}$ & $143.01^{\mathrm{b}}$ & $143.00^{\mathrm{b}}$ & 5.31 \\
\hline Sulfur (mEq/L) & $180.76^{\mathrm{a}}$ & $180.77^{\mathrm{a}}$ & $154.11^{\mathrm{b}}$ & $154.12^{\mathrm{b}}$ & 1.51 \\
\hline \multicolumn{6}{|l|}{ Blood $^{3}$} \\
\hline $\mathrm{pH}$ & $7.36^{\mathrm{b}}$ & $7.37^{\mathrm{b}}$ & $7.40^{\mathrm{a}}$ & $7.39^{\mathrm{a}}$ & 0.004 \\
\hline Calcium (mg/d1) & $9.07^{\mathrm{a}}$ & $9.23^{\mathrm{a}}$ & $8.18^{\mathrm{b}}$ & $8.29^{\mathrm{b}}$ & 0.001 \\
\hline Magnesium (mg/d1) & $2.21^{\mathrm{a}}$ & $2.23^{\mathrm{a}}$ & $2.04^{\mathrm{b}}$ & $2.08^{\mathrm{b}}$ & 0.13 \\
\hline Phosphorus (mg/d1) & 6.48 & 6.49 & 6.47 & 6.48 & 0.03 \\
\hline Sodium $(\mathrm{mEq} / \mathrm{L})$ & 136.99 & 137.31 & 138.22 & 138.36 & 0.86 \\
\hline Potassium (mEq/L) & 4.08 & 4.10 & 4.13 & 4.16 & 0.03 \\
\hline Chloride (mEq/L) & $94.69^{\mathrm{a}}$ & $94.69^{\mathrm{a}}$ & $93.56^{\mathrm{b}}$ & $93.56^{\mathrm{b}}$ & 0.21 \\
\hline \multirow[t]{3}{*}{ Sulfur $(\mathrm{mEq} / \mathrm{L})$} & $1.54^{\mathrm{a}}$ & $1.55^{\mathrm{a}}$ & $1.48^{\mathrm{b}}$ & $1.48^{\mathrm{b}}$ & 0.06 \\
\hline & \multicolumn{4}{|c|}{ Days to calving } & \multirow{2}{*}{ SEM } \\
\hline & 15 & 10 & 5 & 2 & \\
\hline Dry matter intake $(-100)$ & 12.02 & 11.32 & 9.73 & 9.05 & 0.08 \\
\hline Dry matter intake $(+100)$ & 12.47 & 12.57 & 10.95 & 9.98 & 0.11 \\
\hline
\end{tabular}

${ }^{\mathrm{a}-\mathrm{b}}$ Means within a row with different superscrips are different $(\mathrm{p}<0.05)$.

${ }^{1}$ Calculated dietary cation-anion difference $(\mathrm{Na}+\mathrm{K}+0.15 \mathrm{Ca}+0.15 \mathrm{Mg})-(\mathrm{Cl}+0.6 \mathrm{~S}+0.5 \mathrm{P})$.

${ }^{2}$ Samples were collected on $\mathrm{d} 2$ and 12 prepartum. ${ }^{3}$ Samples were collected on $\mathrm{d} 2$ and 12 prepartum.

Table 3. The effect of dietary cation-anion difference on plasma calcium concentration postpartum

\begin{tabular}{|c|c|c|c|c|}
\hline \multirow{2}{*}{ Calcium* } & & \multicolumn{3}{|c|}{ Day } \\
\hline & & 1 & 2 & 12 \\
\hline \multicolumn{5}{|l|}{ Group $^{1}$} \\
\hline 1 & & $8.28^{\mathrm{a}}$ & 9.12 & 9.30 \\
\hline 2 & & $8.27^{\mathrm{a}}$ & 9.08 & 9.29 \\
\hline 3 & & $7.79^{b}$ & 9.10 & 9.29 \\
\hline 4 & & $7.80^{\mathrm{b}}$ & 9.05 & 9.28 \\
\hline SEM & & 0.08 & 0.16 & 0.13 \\
\hline \multirow[t]{2}{*}{ Balance $1^{2}$} & -100 & $8.27^{\mathrm{a}}$ & 9.10 & 9.29 \\
\hline & +100 & $7.79^{\mathrm{b}}$ & 9.08 & 9.28 \\
\hline \multirow[t]{2}{*}{ Balance $2^{3}$} & +400 & 8.034 & 9.11 & 9.29 \\
\hline & +200 & 8.033 & 9.07 & 9.28 \\
\hline SEM & & 0.05 & 0.11 & 0.09 \\
\hline
\end{tabular}

* Calcium $(\mathrm{mg} / \mathrm{dl})$.

${ }^{1}$ In all of tables; Group 1: cows that received prepartum diet with -100 DCAD and postpartum diet with +400 DCAD, group 2: cows that received prepartum diet with -100 DCAD and postpartum diet with +200 DCAD, group 3: cows that received prepartum diet with +100 DCAD and postpartum diet with +400 DCAD, group 4: cows that received prepartum diet with +100 DCAD and postpartum diet with +200 DCAD.

${ }^{2}$ In all of tables; prepartum dietary cation-anion difference levels $(-100$ and $+100 \mathrm{mEq} / \mathrm{kg} \mathrm{DM})$.

${ }^{3}$ In all of tables; postpartum dietary cation-anion difference levels ( +400 and $\left.+200 \mathrm{mEq} / \mathrm{kg} \mathrm{DM}\right)$. 
Table 4. The effect of dietary cation-anion difference on plasma minerals concentrations in postpartum period

\begin{tabular}{|c|c|c|c|c|c|c|c|}
\hline & & \multicolumn{6}{|c|}{ Mineral* } \\
\hline & & $\mathrm{Na}$ & $\mathrm{K}$ & $\mathrm{Mg}$ & $\mathrm{P}$ & $\mathrm{Cl}$ & $\mathrm{S}$ \\
\hline \multicolumn{8}{|l|}{ Group } \\
\hline 1 & & $143.56^{\mathrm{a}}$ & $4.55^{\mathrm{a}}$ & 2.31 & 6.48 & 93.07 & 1.42 \\
\hline 2 & & $138.71^{b}$ & $4.24^{\mathrm{b}}$ & 2.28 & 6.42 & 93.44 & 1.43 \\
\hline 3 & & $143.56^{\mathrm{a}}$ & $4.55^{\mathrm{a}}$ & 2.28 & 6.48 & 93.08 & 1.42 \\
\hline 4 & & $138.71^{\mathrm{b}}$ & $4.23^{\mathrm{b}}$ & 2.26 & 6.43 & 93.44 & 1.42 \\
\hline SEM & & 0.39 & 0.04 & 0.05 & 0.07 & 0.50 & 0.03 \\
\hline \multirow[t]{2}{*}{ Balance 1} & -100 & 141.61 & 4.40 & 2.29 & 6.45 & 93.26 & 1.43 \\
\hline & +100 & 141.13 & 4.39 & 2.12 & 6.64 & 93.26 & 1.42 \\
\hline \multirow[t]{2}{*}{ Balance 2} & +400 & $143.56^{\mathrm{a}}$ & $4.55^{\mathrm{a}}$ & 2.29 & 6.48 & 93.07 & 1.42 \\
\hline & +200 & $138.71^{b}$ & $4.24^{\mathrm{b}}$ & 2.27 & 6.42 & 93.44 & 1.43 \\
\hline SEM & & 0.27 & 0.03 & 0.03 & 0.05 & 0.35 & 0.02 \\
\hline
\end{tabular}

* $\mathrm{Na}(\mathrm{mEq} / \mathrm{L}), \mathrm{K}(\mathrm{mEq} / \mathrm{L}), \mathrm{Mg}(\mathrm{mg} / \mathrm{dl}), \mathrm{P}(\mathrm{mg} / \mathrm{dl}), \mathrm{Cl}(\mathrm{mEq} / \mathrm{L}), \mathrm{S}(\mathrm{mEq} / \mathrm{L})$.

Table 5. Effect of dietary cation-anion difference on urine minerals concentrations in postpartum period

\begin{tabular}{|c|c|c|c|c|c|c|c|c|}
\hline & & \multicolumn{7}{|c|}{ Mineral* } \\
\hline & & $\mathrm{Na}$ & $\mathrm{K}$ & $\mathrm{Ca}$ & $\mathrm{Mg}$ & $\mathrm{P}$ & $\mathrm{Cl}$ & $\mathrm{S}$ \\
\hline \multicolumn{9}{|l|}{ Group } \\
\hline 1 & & 102.44 & $232.24^{\mathrm{a}}$ & $3.43^{\mathrm{b}}$ & 23.95 & $2.36^{\mathrm{b}}$ & $52.23^{\mathrm{b}}$ & $218.54^{b}$ \\
\hline 2 & & 95.82 & $203.30^{\mathrm{b}}$ & $4.73^{\mathrm{a}}$ & 27.74 & $3.06^{\mathrm{a}}$ & $57.92^{\mathrm{a}}$ & $235.51^{\mathrm{a}}$ \\
\hline 3 & & 102.58 & $234.22^{\mathrm{a}}$ & $3.46^{\mathrm{b}}$ & 24.13 & $2.37^{\mathrm{b}}$ & $51.93^{\mathrm{b}}$ & $218.52^{\mathrm{b}}$ \\
\hline 4 & & 97.03 & $203.29^{\mathrm{b}}$ & $4.72^{\mathrm{a}}$ & 27.94 & $3.15^{\mathrm{a}}$ & $57.72^{\mathrm{a}}$ & $235.50^{\mathrm{a}}$ \\
\hline SEM & & 2.5 & 1.62 & 0.29 & 1.9 & 1.6 & 1.46 & 3.55 \\
\hline \multirow[t]{2}{*}{ Balance 1} & -100 & 98.86 & 217.77 & 4.08 & 25.85 & 2.71 & 55.07 & 227.01 \\
\hline & +100 & 99.80 & 218.76 & 4.09 & 26.03 & 2.76 & 54.83 & 227.02 \\
\hline \multirow[t]{2}{*}{ Balance 2} & +400 & $102.51^{\mathrm{a}}$ & $233.23^{\mathrm{a}}$ & $3.45^{\mathrm{b}}$ & 24.04 & $2.36^{\mathrm{b}}$ & $52.08^{\mathrm{b}}$ & $218.53^{\mathrm{b}}$ \\
\hline & +200 & $96.15^{\mathrm{b}}$ & $203.30^{\mathrm{b}}$ & $4.73^{\mathrm{a}}$ & 27.84 & $3.10^{\mathrm{a}}$ & $57.82^{\mathrm{a}}$ & $235.51^{\mathrm{a}}$ \\
\hline \multicolumn{9}{|l|}{$p$ value } \\
\hline SEM & & 1.77 & 1.15 & 0.21 & 1.34 & 1.13 & 1.03 & 2.51 \\
\hline
\end{tabular}

* $\mathrm{Na}(\mathrm{mEq} / \mathrm{L}), \mathrm{K}(\mathrm{mEq} / \mathrm{L}), \mathrm{Ca}(\mathrm{mEq} / \mathrm{L}), \mathrm{Mg}(\mathrm{mEq} / \mathrm{L}), \mathrm{P}(\mathrm{mg} / \mathrm{dl}), \mathrm{Cl}(\mathrm{mEq} / \mathrm{L}), \mathrm{S}(\mathrm{mEq} / \mathrm{L})$.

Table 6. Effect of dietary cation-anion difference on blood and urine $\mathrm{pH}$, milk production and milk compositions in postpartum period

\begin{tabular}{|c|c|c|c|c|c|c|c|c|c|c|}
\hline Group & & $\mathrm{B}-\mathrm{pH}^{1}$ & U-pH ${ }^{2}$ & Milk yield & $4 \% \mathrm{FCM}$ & Fat & Protein & SNF & TS & Lactose \\
\hline 1 & & $7.45^{\mathrm{a}}$ & $8.32^{\mathrm{a}}$ & 31.71 & $30.93^{\mathrm{a}}$ & $4.01^{\mathrm{a}}$ & 2.94 & 8.40 & $13.22^{\mathrm{a}}$ & 4.63 \\
\hline 2 & & $7.42^{\mathrm{b}}$ & $8.26^{\mathrm{b}}$ & 30.08 & $26.58^{b c}$ & $3.62^{\mathrm{ab}}$ & 2.82 & 8.35 & $11.40^{\mathrm{c}}$ & 4.63 \\
\hline 3 & & $7.44^{\mathrm{a}}$ & $8.33^{\mathrm{a}}$ & 30.37 & $28.45^{\mathrm{ab}}$ & $3.81^{\mathrm{a}}$ & 3.04 & 8.43 & $12.50^{\mathrm{b}}$ & 4.59 \\
\hline 4 & & $7.42^{\mathrm{b}}$ & $8.27^{\mathrm{b}}$ & 29.54 & $24.41^{\mathrm{c}}$ & $3.16^{\mathrm{b}}$ & 2.88 & 8.36 & $11.82^{\mathrm{c}}$ & 4.62 \\
\hline SEM & & 0.0003 & 0.006 & 0.61 & 1.04 & 0.17 & 0.11 & 0.15 & 0.21 & 0.005 \\
\hline \multirow[t]{2}{*}{ Balance 1} & -100 & 7.43 & 8.29 & 30.89 & $28.75^{\mathrm{a}}$ & 3.81 & 2.88 & 8.38 & 12.31 & 4.63 \\
\hline & +100 & 7.43 & 8.30 & 29.96 & $26.54^{\mathrm{b}}$ & 3.48 & 2.96 & 8.40 & 12.16 & 4.61 \\
\hline \multirow[t]{2}{*}{ Balance 2} & +400 & $7.45^{\mathrm{a}}$ & $8.33^{\mathrm{a}}$ & 31.04 & $29.69^{\mathrm{a}}$ & $3.91^{\mathrm{a}}$ & 2.99 & 8.42 & $12.86^{\mathrm{a}}$ & 4.61 \\
\hline & +200 & $7.42^{\mathrm{b}}$ & $8.26^{\mathrm{b}}$ & 29.81 & $25.49^{\mathrm{b}}$ & $3.39^{\mathrm{b}}$ & 2.85 & 8.36 & $11.61^{\mathrm{b}}$ & 4.62 \\
\hline SEM & & 0.0002 & 0.004 & 0.43 & 0.73 & 0.12 & 0.08 & 0.10 & 0.15 & 0.05 \\
\hline
\end{tabular}

${ }^{\mathrm{a}-\mathrm{c}}$ Means within a column with different superscrips are significantly different $(\mathrm{p}<0.05) .{ }^{1} \mathrm{pH}$ blood. ${ }^{2} \mathrm{pH}$ Urinary. 


\section{Dry matter intake and lactation performance}

Cows fed -100 DCAD had lower $(\mathrm{p}<0.01)$ DMI during $15,10,5$ and $2 \mathrm{~d}$ prior to parturition (Table 2). Cows fed +400 DCAD consumed more DM than those fed +200 DCAD at 2, 4, 5 and 9 wk postpartum (Table 7). In addition, cows fed -100 DCAD prepartum had higher DMI at wk 1 postpartum (Table 7) and 4\% FCM yield (Table 6) than cows fed +100 DCAD $(p<0.01)$. Milk yield did not differ among treatments. However, milk fat and total solid percentages and 4\% FCM yield increased for cows fed +400 DCAD in early lactation period.

\section{Health status}

Milk fever case was not detected in this study. Four episodes of hypocalcemia were observed in cows fed +100 DCAD diet, but no hypocalcemia occurred in the cows fed -100 DCAD diet. There were no significant differences in the occurrence of retained placenta, metritis, endometritis, ketosis, mastitis and udder edema due to DCAD treatments. However, the prevalence of disorders tended to be lower in the group of cows fed -100 DCAD diet than the positive DCAD diet (Table 8).

\section{DISCUSSION}

\section{Fluid acid-base balance}

The kidney can efficiently eliminate excess anions from the blood, thus addition of anionic salts induces a sharp reduction in urinary $\mathrm{pH}$. The negative $\mathrm{DCAD}$ diet may have overcome the capacity of kidneys to excrete sufficient $\mathrm{H}^{+}$to maintain a constant blood $\mathrm{pH}$, resulting in a slight systemic acidosis (Tucker et al., 1992). It is well documented (Joyce et al., 1997; Pehrson et al., 1999; Shahzad et al., 2008) that increased dietary anions $(\mathrm{Cl}$ and $\mathrm{S})$ decreased the urine $\mathrm{pH}$. Monitoring the $\mathrm{pH}$ of urine may be considered a sensitive method for assessing the acid-base balance in extracellular
Table 8. The effect of dietary cation-anion difference on health status

\begin{tabular}{|c|c|c|c|c|}
\hline \multirow{3}{*}{$\begin{array}{l}\text { Prepartum } \\
\text { Postpartum } \\
\end{array}$} & \multicolumn{4}{|c|}{$\mathrm{DCAD}^{*}(\mathrm{mEq} / \mathrm{kg} \mathrm{DM})$} \\
\hline & \multicolumn{2}{|c|}{-100} & \multicolumn{2}{|c|}{+100} \\
\hline & +200 & +400 & +200 & +400 \\
\hline Milk fever & $0 / 6$ & $0 / 6$ & $0 / 6$ & $0 / 6$ \\
\hline Hypocalcemia & $0 / 6$ & $0 / 6$ & $2 / 6$ & $2 / 6$ \\
\hline Retained placenta & $0 / 6$ & $0 / 6$ & $3 / 6$ & $1 / 6$ \\
\hline Udder edema & $0 / 6$ & $0 / 6$ & $1 / 6$ & $3 / 6$ \\
\hline Ketosis & $0 / 6$ & $0 / 6$ & $1 / 6$ & $0 / 6$ \\
\hline Mastitis & $1 / 6$ & $0 / 6$ & $3 / 6$ & $4 / 6$ \\
\hline Metritis & $0 / 6$ & $0 / 6$ & $2 / 6$ & $0 / 6$ \\
\hline Endometritis & $0 / 6$ & $0 / 6$ & $2 / 6$ & $0 / 6$ \\
\hline No. of total disorder & 1 & 0 & 14 & 10 \\
\hline
\end{tabular}

fluids (Seifi et al., 2004). Results of the current study showed an incidence of a mild metabolic acidosis as reported by Vagnoni and Oetzel (1998). Similar results have been observed in previous studies for dairy cows (West et al., 1991; Moore et al., 2000; Charbanuae et al., 2006). Thus, DCAD has been shown to be associated with fluid acid-base balance. Spanghero (2004) found that DCAD was associated with urinary $\mathrm{pH}\left(\mathrm{r}^{2}=0.81\right)$ as also noticed by Liesegang et al. (2007). A higher urine $\mathrm{pH}$ has also been reported with increased cation $(\mathrm{Na}$ or $\mathrm{K}$ ) of diet (Waterman et al., 1991). Alteration in urine $\mathrm{pH}$ reflected alteration in blood $\mathrm{pH}$ and kidneys played a vital role to minimize this change by making the urine $\mathrm{pH}$ alkaline, by excreting more $\mathrm{HCO}_{3}{ }^{-}$and conserving $\mathrm{H}^{+}$(Roche et al., 2003).

\section{Plasma mineral concentration}

A slight variation in prepartum plasma $\mathrm{Na}$ and $\mathrm{K}$ might be attributed to dietary alteration of these minerals as excess

Table 7. The effect of dietary cation-anion difference on dry matter intake in postpartum period

\begin{tabular}{|c|c|c|c|c|c|c|c|c|c|c|}
\hline \multirow{2}{*}{ Group } & & \multicolumn{9}{|c|}{ Week } \\
\hline & & 1 & 2 & 3 & 4 & 5 & 6 & 7 & 8 & 9 \\
\hline 1 & & $14.36^{\mathrm{a}}$ & $15.00^{\mathrm{a}}$ & 16.78 & $18.57^{\mathrm{a}}$ & $20.25^{\mathrm{a}}$ & 21.35 & $22.42^{\mathrm{ab}}$ & 23.11 & $23.61^{a}$ \\
\hline 2 & & $14.30^{\mathrm{a}}$ & $14.43^{b}$ & 16.35 & $18.25^{b}$ & $19.47^{\mathrm{b}}$ & 20.88 & $21.96^{\mathrm{b}}$ & 22.86 & $23.10^{b}$ \\
\hline 3 & & $13.18^{\mathrm{b}}$ & $14.91^{\mathrm{a}}$ & 16.76 & $18.65^{\mathrm{a}}$ & $19.93^{\mathrm{ab}}$ & 21.22 & $22.78^{\mathrm{a}}$ & 23.06 & $23.58^{\mathrm{a}}$ \\
\hline 4 & & $13.20^{\mathrm{b}}$ & $14.45^{\mathrm{b}}$ & 16.45 & $18.12^{b}$ & $19.52^{b}$ & 21.23 & $22.63^{\mathrm{ab}}$ & 23.06 & $23.00^{\mathrm{b}}$ \\
\hline SEM & & 0.16 & 0.12 & 0.14 & 0.08 & 0.18 & 0.20 & 0.24 & 0.12 & 0.14 \\
\hline \multirow[t]{2}{*}{ Balance $1^{1}$} & -100 & $14.33^{\mathrm{a}}$ & 14.71 & 16.57 & 18.41 & 19.85 & 21.12 & $22.19^{b}$ & 22.99 & 23.36 \\
\hline & +100 & $13.91^{\mathrm{b}}$ & 14.68 & 16.61 & 18.38 & 19.72 & 21.22 & $22.71^{\mathrm{a}}$ & 23.06 & 23.29 \\
\hline \multirow[t]{2}{*}{ Balance $2^{2}$} & +400 & 13.77 & $14.96^{\mathrm{a}}$ & $16.77^{\mathrm{a}}$ & $18.61^{\mathrm{a}}$ & $20.09^{\mathrm{a}}$ & 21.28 & 22.60 & 23.09 & $23.60^{\mathrm{a}}$ \\
\hline & +200 & 13.75 & $14.44^{\mathrm{b}}$ & $16.40^{\mathrm{b}}$ & $18.18^{b}$ & $19.49^{b}$ & 21.05 & 22.30 & 22.96 & $23.05^{\mathrm{b}}$ \\
\hline SEM & & 0.88 & 0.01 & 0.02 & 0.01 & 0.03 & 0.29 & 0.23 & 0.32 & 0.01 \\
\hline
\end{tabular}

\footnotetext{
${ }^{1}$ Prepartum dietary cation-anion difference levels $(-100$ and $+100 \mathrm{mEq} / \mathrm{kg} \mathrm{DM})$.
}

${ }^{2}$ Postpartum dietary cation-anion difference levels $(+400$ and $+200 \mathrm{mEq} / \mathrm{kg} \mathrm{DM})$. 
dietary $\mathrm{Na}$ and $\mathrm{K}$ were excreted through kidney ( $\mathrm{Hu}$ and Murphy, 2004). Similar results were reported by West et al. (1991) who stated that increased DCAD level $(-116$ to +312 $\mathrm{mEq} / \mathrm{kg} \mathrm{DM})$ did not affect the plasma sodium and potassium concentrations significantly.

The increased plasma Ca level for cows consuming -100 DCAD diet compared to those receiving +100 DCAD diet might be due to mild metabolic acidosis induced by negative DCAD concentration. Bones act as a major reservoir of buffers for acid-base control of body fluids. When animals are placed on acidifying diets, the blood $\mathrm{pH}$ decreases. Frick et al. (2009) concluded that an acidic extracellular $\mathrm{pH}$ increases osteoclastic bone resorption which may result in increased plasma $\mathrm{Ca}$ concentrations.

The nadir of plasma $\mathrm{Ca}$ observed on $\mathrm{d} 1$ may be due to the highly increased blood $\mathrm{Ca}$ demand for colostrum production. Kume et al. (2001) reported negative retention after calving. Similar findings were reported by Charbonneau et al. (2006); Lean et al. (2006) and Moore et al. (2000). The increased plasma Mg concentration in cows fed -100 DCAD may result from their higher $\mathrm{Mg}$ intake in close-up diets. Similar results were reported by Joyce et al. (1997); Lean et al. (2006) and Li et al. (2008). There was no significant effect of prepartum DCAD levels on serum phosphorus which is consistent with other reports (Shahzad et al., 2008; Wu et al., 2008). An increase in plasma chloride and sulphur concentrations by decreasing the DCAD level to $-100 \mathrm{mEq} / \mathrm{kg}$ of DM is also supported by Roche (1999) and Tucker et al. (1988) who reported a linear increase in plasma $\mathrm{Cl}$ and $\mathrm{S}$ concentration with decreased DCAD level and it may be due to dietary concentrations in their experiments. In early lactation, there was no difference between treatments regarding $\mathrm{Ca}, \mathrm{Mg}, \mathrm{P}, \mathrm{Cl}$ and $\mathrm{S}$ contents of plasma. This finding is supported by Delaquis and Block (1995a) who reported that mineral concentrations were not significantly affected by dietary DCAD levels. Increased plasma $\mathrm{Na}$ and $\mathrm{K}$ concentrations in cows fed +400 $\mathrm{mEq} / \mathrm{kg} \mathrm{DM}$ are consistent with the findings of Roche et al. (2005) who observed a linear increase in plasma $\mathrm{Na}$ and $\mathrm{K}$ concentrations of early lactating cows as ration DCAD increased.

\section{Urine mineral concentration}

The findings of the present study for urine mineral concentrations were in line with Tucker et al. $(1988 ; 1992)$ who reported increased excretions of $\mathrm{Na}$ and $\mathrm{K}$ as the DCAD level increased from -20 to $10 \mathrm{mEq} / 100 \mathrm{~g}$ of DM, while $\mathrm{Cl}$ and $\mathrm{S}$ excretions decreased. This increase in $\mathrm{Na}$ and $\mathrm{K}$ concentrations with high DCAD diet is due to the fact that high DCAD diet contains higher contents of these minerals and urine composition is closely associated with diet composition (West et al., 1991).
The present findings are also consistent with West et al. (1992) who reported increased urinary $\mathrm{Na}$ and $\mathrm{K}$ concentrations at high DCAD levels while its reverse was true for $\mathrm{Cl}$ concentration. Although, dietary concentrations of $\mathrm{Cl}$ and $\mathrm{S}$ were similar between treatments, their urinary concentrations were higher in cows fed $+200 \mathrm{mEq} / \mathrm{kg}$ of DM. This is in line with Delaquis and Block (1995b) who recorded increased urinary $\mathrm{S}$ in early lactating cows with decreased DCAD level. High dietary $\mathrm{Cl}$ content of cows fed -100 DCAD diet caused the kidneys to excrete more $\mathrm{Cl}$ in urine to maintain normal blood $\mathrm{pH}$ but in spite of that a slight metabolic acidosis was experienced, as was evident from altered prepartum blood $\mathrm{pH}$. Similar findings were reported by West et al. (1991; 1992) who reported increased urinary $\mathrm{Cl}$ by decreasing the DCAD level. In pre-calving, increased urinary $\mathrm{S}$ by decreasing the level of DCAD might be attributed to dietary concentrations. So, more S intake may result in more plasma and urinary concentrations and vice versa (Takagi and Block, 1991). Increased urinary Ca excretion in cows fed -100 DCAD might be due to a slight metabolic acidosis, induced by negative DCAD diet. The pre-calving reduction in systemic $\mathrm{pH}$ was associated with an increased urinary output of $\mathrm{Ca}$ and this metabolic acidosis might have increased $\mathrm{Ca}$ resorption from bones and intestinal $\mathrm{Ca}$ absorption (Roche et al., 2003) due to increased synthesis of $1,25(\mathrm{OH})_{2} \mathrm{D}_{3}$ (Goff et al., 1991).

It is also reported that ruminants' kidneys are highly sensitive to blood cation-anion difference and increase the excretion of $\mathrm{Ca}$ during acidosis, independent of the hormonal action usually associated with $\mathrm{Ca}$ metabolism (Stacy and Wilson, 1970). Increased urinary Mg in cows fed -100 DCAD diet might be attributed to improved absorption because $\mathrm{Mg}$ absorption increased as DCAD level decreased. Moreover, increased urinary $\mathrm{Mg}$ concentration might be an indirect indicator of improved $1,25(\mathrm{OH})_{2} \quad \mathrm{D}_{3}$ synthesis, because $\mathrm{Mg}$ is utilized when $25(\mathrm{OH}) \mathrm{D}_{3}$ is converted to its active form $1,25(\mathrm{OH})_{2} \mathrm{D}_{3}$, and during slight metabolic acidosis this process works more efficiently and thereby releasing more $\mathrm{Mg}$ in urine (Chamberlain and Wilkinson, 1996). Similar findings are reported by Gaynor et al. (1989); Lean et al. (2006); Oetzel et al. (1988); and Wu et al. (2008). In prepartum, urinary $P$ concentration was unaffected by either DCAD treatments or time. This is supported by finding of Delaquis and Block (1995a) and Joyce et al. (1997). Postpartum, urinary P increased in cows fed +200 DCAD diet compared to those fed +400 DCAD diet, which is in agreement with Delaquis and Block (1995b) who reported increased urinary $\mathrm{P}$ at low DCAD compared to those fed high DCAD diets.

\section{Dry matter intake and lactation performance}

Decreased DMI in cows fed negative DCAD diet during 
the days prior to parturition might be attributed to low rumen $\mathrm{pH}$ demonstrated by Tucker et al. (1991). The finding confirms results of previous researches (Block, 1984; Chan et al., 2006; Charbannuae et al., 2006) that showed anionic salts in a TMR decrease DMI prepartum. Vagnoni and Oetzel (1998) speculated that the reduction in feed intake for non-lactating dairy cows is likely caused by the acidogenic response to anion supplementation, rather than inherent poor palatability of supplemental acidogenic salts. This was supported by the observation that the more acidogenic mixtures caused the greatest feed intake depression of nonlactating dairy cows in a comparison among several different anion sources.

Increased DMI by cows fed +400 DCAD might be attributed to increased blood $\mathrm{HCO}_{3}{ }^{-}$, acid-base balance (Sanchez and Beede, 1994) and rumen pH (Tucker et al., 1991). Increased DMI with increasing the DCAD has also been reported by many researchers (Tucker et al., 1991; West et al., 1991; Hu et al., 2007:2007a). However, the present results did not agree with Chan et al. (2005) who reported that increasing DCAD from 20 to $50 \mathrm{mEq} / 100 \mathrm{~g}$ of DM had no effect on DMI in cows from 0 to $42 \mathrm{~d}$ postpartum. Buffer addition increased feed consumption in some studies (Kilmer et al., 1981). Moreover, by wk 1 postpartum, cows fed -100 DCAD diet had higher DMI than cows fed +100 DCAD diet. Improvements in Ca status apparently overcome any detrimental effects of reduced prepartum DMI (Joyce et al., 1997). Milk yield did not differ among treatments. Similar results have been observed in previous studies for dairy cows (Apper-Bossard et al., 2006; Hu et al., 2007b; Wildman et al., 2007; Wu et al., 2008).

Increased milk fat and TS percentages and also $4 \%$ FCM yield in cows fed +400 DCAD diet may be attributed to additional ruminal buffering provided by +400 DCAD diets. These results are consistent with those of others (Roche et al., 2005; $\mathrm{Hu}$ et al., 2007a) that have demonstrated a positive relationship between DCAD and milk fat concentration. Numerous studies have shown that addition of dietary buffers such as $\mathrm{NaHCO}_{3}$ and $\mathrm{K}_{2} \mathrm{CO}_{3}$ increase milk fat percentage, especially when depressed milk fat occurred (Hu et al., 2007a). The effect of buffers on milk fat production is probably mediated via the rumen. Milk fat percentage is positively related to ruminal $\mathrm{pH}$ (Allen, 1997). Higher rumen $\mathrm{pH}$ has been reported to decrease the concentration of trans fatty acids in the rumen (Wildman et al., 2007). West et al. (1992) reported an increase in the milk fat percentage by increasing DCAD, without any effect on milk yield. Conversely, some studies have reported an increase in milk yield without changes in milk fat percentage (Tucker et al., 1991; West et al., 1991). Unaltered protein, lactose and SNF percentages due to alteration in DCAD are in concordance with other studies (Tucker et al., 1988; West et al., 1992).

\section{Health status}

There were no cases of milk fever in this study. Present results showed the expulsion time of placenta after parturition was lower in animals fed anionic diets which confirmed the results of Goff and Horst (1998). The results of Joyce et al. (1997) showed, in cows fed low DCAD diets, incidence of retained placenta was zero, but $\mathrm{Hu}$ et al. (2007a) observed diets with low DCAD did not alter the incidence of metabolic disorders. Overall, the reduced DCAD diet could improve periparturient dairy cow health.

\section{IMPLICATIONS}

The results of this study clearly show the role of DCAD in regulating DMI and blood acid-base status. Supplementation of diets in the last $3 \mathrm{wk}$ prepartum with anionic salts mixture at a rate sufficient to decrease DCAD dietary may benefit blood calcium homeostasis and tend to increase 4\% FCM production and DMI in first week during postpartum and also improve health status. Thus, an input of -100 DCAD at close-up period followed by +400 DCAD at early lactation rations is recommended. Changes in urinary and blood $\mathrm{pH}$ and excretion of minerals were consistent with changes in DCAD. Further larger-scale and longer-term trials are needed to confirm the data presented here.

\section{ACKNOWLEDGEMENTS}

This research was performed in Ramdoosh Dairy Company, Shahriyar, Tehran, Iran.

\section{REFERENCES}

Allen, M. C. 1997. Relationship between fermentation acid production in the rumen and the requirement for physically effective fiber. J. Dairy Sci. 80:1447-1462.

AOAC. 1990. Official methods of analysis. 15th edn. Association of Official Analytical Chemists, Arlington, Virginia, USA.

Apper-Bossard, E., J. L. Peyraud, P. Faverdin and F. Meschy. 2006. Changing dietary cation anion difference for dairy cows fed with two contrasting levels of concentrate in diets. J. Dairy Sci. 89:749-760.

Beede, D. K. 1995. Practical application of cation-anion difference in dairy rations. In Proceedings of the 1995 Maryland Nutrition. Conf For Feed Manuf., Univ. Maryland, College Park, MD. pp. 80-89.

Beede, D. K., C. A. Risco, G. A. Donovan, C. Wang, L. F. Archbald and W. K. Sanchez. 1992. Nutritional management of the late pregnant dry cow with particular reference to dietary cation-anion difference and calcium supplementation. 
In Proceedings of 24th Annual Convention American Association of Bovine Practitioners, Orlando, FL Frontier Printers, Stillwater, OK. p. 51.

Block, E. 1984. Manipulating dietary anions and cations for prepartum dairy cows to reduce incidence of milk fever. J. Dairy Sci. 67:2939-2948.

Chamberlaine, A. T. and J. M. Wilconson. 1996. Minerals and vitamins in feeding the dairy cow. Chalcombe Publications, Great Britain. pp. 79-94.

Chan, P. S., J. W. West and J. K. Bernard. 2006. Effect of prepartum dietary calcium on intake and serum and urinary mineral concentrations of cows. J. Dairy Sci. 89:704-713.

Chan, P. S., J. K. West, J. K. Bernard and J. M. Fernandes. 2005. Effects of dietary cation-anion difference on intake, milk yield, and blood components of the early lactation cow. J. Dairy Sci. 88:4384-4392.

Charbanneau, E., D. Pellerin and G. R. Oetzel. 2006. Impact of lowering dietary cation-anion difference in nonlactating dairy cows: A meta-analysis. J. Dairy Sci. 89:537-548.

Curtis, C. R., H. N. Erb, C. J. Sniffen and R. D. Smith. 1983. Epidemiology of parturient paresis: predisposing factors with emphasis on dry cow feeding and management. J. Dairy Sci. 67:817-825.

DeGaris, P. J. and I. J. Lean. 2008. Milk fever in dairy cows: A review of pathophysiology and control principles. Vet. J. 176:58-69.

Delaquis, A. M. and E. Block. 1995a. Acid base status, renal function, water, and macromineral metabolism of dry cows fed diets differing in cation anion difference. J. Dairy Sci. 78:604619.

Delaquis, A. M. and E. Block. 1995b. The effects of changing ration ingredients on acid base status, renal function, and macromineral metabolism. J. Dairy Sci. 78:2024-2039.

Frick, K. K., N. S. Krieger, K. Nehrke and D. A. Bushinsky. 2009. Metabolic acidosis increases intracellular calcium in bone cells through activation of the proton receptor OGR1. J. Bone Miner. Res. 24:305-313.

Gaynor, P. J., F. J. Mueller, J. K. Miller, N. Ramsey, J. P. Goff and R. L. Horst. 1989. Parturient hypocalcemia in Jersey cows fed alfalfa haylage based diets with different cation to anion rations. J. Dairy Sci. 72:2525-2531.

Goff, J. P., R. L. Horst, F. J. Mueller, J. K. Miller, J. A. Kiess and H. H. Dowlen. 1991. Addition of chloride to a prepartal diet high in cations increase 1,25- dihydroxyvitamin D response to hypocalcemia preventing milk fever. J. Dairy Sci. 74:38633871.

Goff, J. P. and R. L. Horst. 1997. Physiological changes at parturition and their relationship to metabolic disorders. J. Dairy Sci. 80:1260-1268.

Goff, J. P. and R. L. Horst. 1998. Use of hydrochloric acid as a source of anions for prevention of milk fever. J. Dairy Sci. $81: 2874-2880$

Goff, J. P. 2008. The monitoring, prevention, and treatment of milk fever and subclinical hypocalcemia in dairy cows. Vet. J. 176:50-57.

Grohn, Y. T., H. N. Erb, C. E. McCulloch and H. S. Saloniemi. 1989. Epidemiology of metabolic disorders in dairy cattle: Associations among host characteristics, disease and production. J. Dairy Sci. 72:1876-1885.
Hansen, T. T., R. L. JØjensen and S. Østergaard. 2002. Milk fever control principles: A review. Acta Vet. Scand. 43:1-19.

Horst, R. L., J. P. Goff, T. A. Reinhardt and D. R. Boxton. 1997. Strategies for preventing milk fever in dairy cattle. J. Dairy Sci. 80:1269-1280.

Horst, R. L., J. P. Goff and T. A. Reinhardt. 1994. Calcium and vitamin D metabolism in the dairy cow. J. Dairy Sci. 77:19361951.

Hu, W. and M. R. Murphy. 2004. Dietary cation-anion difference on performance and acid-base status of lactating dairy cows. J. Dairy Sci. 87:2222-2229.

Hu, W., J. R. Limin Kung and M. R.Murphy. 2007. Relationship between dry matter intake and acid-base status of lactating dairy cows as manipulated by dietary cation-anion difference. Anim. Feed Sci. Technol. 136:216-225.

Hu, W., M. R. Murphy, P. D. Constable and E. Block. 2007a. Dietary cation-anion difference and dietary protein effects on performance and acid-base status of dairy cows in early lactation. J. Dairy Sci. 90:3355-3366.

Hu, W., M. R. Murphy, P. D. Constable and E. Block. 2007b. Dietary cation-anion difference effects on performance and acid-base status of dairy cows postpartum. J. Dairy Sci. 90:3367-3375.

Joyce, P. W., W. K. Sanchez and J. P. Goff. 1997. Effect of anionic salts in prepartum diets based on alfalfa. J. Dairy Sci. 80:28662875.

Kelton, D. F., K. D. Lissemore and R. E.Martin. 1998. Recommendations for recording and calculating the incidence of selected clinical diseases of dairy cattle. J. Dairy Sci. 81:2502-2509.

Kilmer, L. H., L. D. Muller and T. J. Snyder. 1981. Addition of sodium bicarbonate to rations of postpartum dairy cows:physiology and metabolic effects. J. Dairy Sci. 64:2357.

Kimura, K., J. P. Goff, M. E. Kehrli and T. A. Reinhardt. 2002. Decreased neutrophil function as a cause of retained placenta in dairy cattle. J. Dairy Sci. 85:544-550.

Kume, S., K. Toharmat, K. Nonaka, T. Oshiat, T. Nakui and J. Ternouth. 2001. Relationship between crude protein and mineral concentrations in alfalfa and value of alfalfa silage as a mineral source for priparturient cows. Anim. Feed Sci. Technol. 93:157-168.

Lean, I. J., P. J. DeGaris, D. M. Mcneil and E. Block. 2006. Hypocalcemia in dairy cows: Meta-analysis and dietary cationanion difference theory revisited. J. Dairy Sci. 89:669-684.

Li, F. C., H. F. Liu and Z. H. Wang. 2008. Effect of dietary cationanion difference on calcium, nitrogen metabolism and relative blood traits of dry Holstein cows. Anim. Feed Sci. Technol. 142:185-191.

Liesegang, A., C. Chiappi, J. Risteli, J. Kessler and H. D. Hess. 2007. Influence of different calcium contents in diets supplemented with anionic salts on bone metabolism in periparturient dairy cows. J. Anim. Physiol. Anim. Nutr. 91:120-129.

Moore, S. J., M. J. Vandehaar, K. Sharma, T. F. Pilbcam, D. K. Beede, F. Bucholtz, J. S. Liesman, R. L. Horst and J. P. Goff. 2000. Effect of altering dietary cation-Anion difference on calcium and energy metabolism in peripartum cows. J. Dairy Sci. 83:2095-2104.

National Research Council. 2001. Nutrient requirements of dairy 
cattle. 7th Ed. National Academy of Sciences, Washington, DC, USA.

Oetzel, G. R., J. D. Olson, C. R. Curtis and M. J. Fettman. 1988. Ammonium chloride and amonium sulfate for prevention of parturient paresis in dairy cows. J. Dairy Sci. 71:3302-3309.

Pehrson, B., C. Svensson, I. Gruvaeus and M. Vrikki. 1999. The influence of acidic diets on the acid-base balance of dry cows and the effect of fertilization on the mineral content of grass. J. Dairy Sci. 82:1310-1316.

Roche, J. R. 1999. Dietary cation-anion difference in pasture-fed dairy cows. Ph.D. Thesis, National University of Ireland.

Roche, J. R., D. Dally, P. Moate, C. Grainger, M. Rath and F. O. Mara. 2003. Dietary cation-anion difference and the health and production of pastured-fed dairy cows 2. Nonlactating periparturient cows. J. Dairy Sci. 86:979-987.

Roche, J. R., S. Peth and J. K. Kay. 2005. Manipulating the dietary cation anion difference via drenching to early lactating dairy cows grazing pasture. J. Dairy Sci. 88:264-276.

Sanchez, W. K. and D. K. Beede. 1994. Interactions of sodium, potassium, and chloride on lactation, acid-base status, and mineral concentrations. J. Dairy Sci. 77:1661-1675.

Sanchez, W. K., D. K. Beede and J. A. Cornell. 1997. Dietary mixtures of sodium bicarbonate, sodium chloride, and potassium chloride: Effects on lactational performance, acidbase status, and mineral metabolism of Holstein cows. J. Dairy Sci. 80:1207-1216.

Sanchez, W. K. 2003.The latest in dietary cation-anion difference (DCAD) Nutrition. In: Proceeding of 43nd Annual Dairy Cattle Day. 26th March, Main Theater, University of California.Davis Campus.

SAS Institute Inc. 2004. SAS/SAT user's guide: Version 9. 2th edn. SAS Institute Inc., Cary, North Carolina.

Seifi, H. A., M. Mohri and J. Kalamati-Zadeh. 2004. Use of prepartum urine $\mathrm{pH}$ to predict the risk of milk fever in dairy cows. Vet. J. 167:281-285.

Shahzad, M. A., M. Sarwar and M. Nisa. 2008. Influence of varying dietary cation anion difference on serum minerals, mineral balance and hypocalcemia in Nili Ravi buffaloes. Livest. Sci. 113:52-61.

Spanghero, M. 2004. Prediction of urinary and blood pH in nonlactating dairy cows fed anionic diets. Anim. Feed Sci. Technol. 116:83-92.
Stacy, B. B. and B. W. Wilson. 1970. Acidosis and hypercalciuria: Renal mechanisms affecting calcium, magnesium and sodium excretion in sheep. J. Physiol. 210:549-564.

Takagi, H. and E. Block. 1991. Effects of reducing dietary cationanion balance on calcium kinetics in sheep. J. Dairy Sci. 74:4225-4237.

Tucker, W. B., G. A. Harrison and R. W. Hemken. 1988. Influence of dietary cation-anion balance on milk, blood, urine, and rumen fluid in lactating dairy cattle. J. Dairy Sci. 71:346-354.

Tucker, W. B., J. F. Hogue. D. F. Waterman, T. S. Swenson, Z. Xin, R. W. Hemken, J. A. Jackson, J. D. Adams and L. J. Spicer. 1991. Role of sulfur and chloride in the dietary cation-anion balance equation for lactating dairy cattle. J. Anim. Sci. 69:1205-1213

Tucker, W. B., J. F. Hogue, G. D. Adams, M. Aslam, I. S. Shin and G. Morgan. 1992. Influence of dietary cation-anion balance during the dry period on the occurrence of parturient paresis in cows fed excess calcium. J. Anim. Sci. 70:1238-1250.

Vagnoni, D. B. and G. R. Oetzel. 1998. Effects of dietary cationanion difference on the acid-base status of dry cows. J. Dairy Sci. 81:1643-1652.

Van Soest, P. J., P. J. Robertson and H. B. Lewis. 1991. Methods of dietary fiber, NDF and non-starch polysaccharides in relation to animal material. J. Dairy Sci. 74:3583-3597.

Waterman, D. F., T. S. Swenson, W. B. Tucker and R. T. Henkin. 1991. Role of magnesium in the dietary cation-anion balance equation for ruminants. J. Dairy Sci. 74:1866-1873.

West, J. K., B. G. Mullinix and T. G. Sandifer. 1991. Changing dietary electrolyte balance for dairy cows in cool and hot environments. J. Dairy Sci. 74:1662.

West, J. W., K. D. Haydon, B. G. Mullinix and T. G. Sandifer. 1992. Dietary cation-anion balance and cation source effects on production and acid-base status of heat stressed cows. J. Dairy Sci. 75:2776.

Wildman, C. D., J. W. West and J. K. Bernard. 2007. Effect of dietary cation-anion difference and dietary crude protein on performance of lactating dairy cows during hot weather. J. Dairy Sci. 90:1842-1850.

Wu, W. X., J. X. Liu, G. Z. Xu and J. A. Ye. 2008. Calcium homeostasis acid-base balance, and health status in periparturient Holstein cows fed diets with low cation-anion difference. Livest. Sci. 117:7-14. 